PROCEEDINGS OF THE

AMERICAN MATHEMATICAL SOCIETY

Volume 128, Number 4, Pages 1221-1229

S 0002-9939(99)05498-2

Article electronically published on October 18, 1999

\title{
THE RESIDUES OF THE RESOLVENT ON DAMEK-RICCI SPACES
}

\author{
R. J. MIATELLO AND C. E. WILL
}

(Communicated by Roe Goodman)

\begin{abstract}
We determine the poles and residues of the resolvent kernel of the Laplacian on a Damek-Ricci space $S$. We show that all poles are simple and the residues define convolution operators of finite rank. This generalizes a result of Guillopé-Zworski for the real hyperbolic $n$-space. If $S$ corresponds to a symmetric space of negative curvature $G / K$, the image of each residue is a $\mathfrak{g}_{c}$-module with a specific highest weight. We compute the dimension by the Weyl dimension formula.
\end{abstract}

\section{Preliminaries}

In this section we will recall some basic notions on $H$-type groups and their canonical solvable extensions, following mainly [2] (see also [1]).

Let $\mathfrak{n}$ be a two-step real nilpotent Lie algebra endowed with an inner product $\langle$,$\rangle such that \mathfrak{n}$ has an orthogonal decomposition $\mathfrak{n}=\mathfrak{z} \oplus \mathfrak{v}$, where $\mathfrak{z}$ is the center of $\mathfrak{n}$ and $[\mathfrak{v}, \mathfrak{v}]=\mathfrak{z}$. If $\mathfrak{n}$ is abelian, we shall use the convention that $\mathfrak{v}=0$ and $\mathfrak{n}=\mathfrak{z}$.

Define a linear mapping $J: \mathfrak{z} \rightarrow \operatorname{End}(\mathfrak{v})$ by

$$
\left\langle J_{Z} X, Y\right\rangle=\langle Z,[X, Y]\rangle
$$

(note that $J_{Z}$ is skew-symmetric). Now $\mathfrak{n}$ is said to be an H-type algebra if for any $Z_{1}, Z_{2} \in \mathfrak{z}$,

$$
J_{Z_{1}} J_{Z_{2}}+J_{Z_{2}} J_{Z_{1}}=-2\left\langle Z_{1}, Z_{2}\right\rangle .
$$

The corresponding $H$-type group is the simply connected Lie group $N$ with Lie algebra $\mathfrak{n}$, endowed with the left-invariant metric induced by the inner product $\langle$, on $\mathfrak{n}$.

Consider the solvable extension, $S=A N$, the semidirect product of $A=\mathbf{R}^{+}$ and $N$, where each $t \in A$ acts on $N$ by $(x, z) \rightarrow\left(t^{\frac{1}{2}} x, t z\right)$.

Let $\mathfrak{s}, \mathfrak{a}$, denote respectively the Lie algebras of $S, A$. Then $\mathfrak{s}=\mathfrak{a} \oplus \mathfrak{n}$ and $\mathfrak{a}=\mathbf{R} H$, where $a d H$ is the derivation of $\mathfrak{n}$ such that $\left.a d H\right|_{\mathfrak{v}}=\frac{1}{2} I$ and $\left.\operatorname{ad} H\right|_{\mathfrak{a}}=I$. Also, $\mathfrak{s}$ carries the inner product extending the one on $\mathfrak{n}$ such that $\|H\|=1,\langle H, \mathfrak{n}\rangle=0 ; S$ carries the induced left-invariant riemannian structure. Furthermore, let $q=\operatorname{dim} \mathfrak{z}$, $p=\operatorname{dim} \mathfrak{v}, n=\operatorname{dim} \mathfrak{s}=p+q+1$ and $Q=\frac{1}{2}(p+2 q)$.

Received by the editors May 27, 1998.

1991 Mathematics Subject Classification. Primary 22E30.

This research was partially supported by Conicet, Conicor, SecytUNC (Córdoba), and I.C.T.P. (Trieste).

(C)2000 American Mathematical Society 
Using coordinates from $\mathfrak{v} \oplus \mathfrak{z} \oplus \mathbf{R}^{+}$, the product on $S$ is expressed as

$$
(X, Z, a)\left(X^{\prime}, Z^{\prime}, a^{\prime}\right)=\left(X+a^{\frac{1}{2}} X^{\prime}, Z+a Z^{\prime}+\frac{1}{2} a^{\frac{1}{2}}\left[X, X^{\prime}\right], a a^{\prime}\right) .
$$

The volume element of the induced left-invariant riemannian metric on $S$ is the left Haar measure

$$
d m=a^{-Q-1} d X d Z d a .
$$

We will use the fact that $S$ can be realized as the unit ball in $\mathfrak{s}$ :

$$
B(\mathfrak{s})=\left\{(X, Z, u):|X|^{2}+|Z|^{2}+u^{2}=1\right\}
$$

via a Cayley type transform $\tilde{C}: S \rightarrow B(\mathfrak{s})$ (see [2], Section 4).

In $B(\mathfrak{s})$ the geodesics through the origin are the diameters and the geodesic distance to the origin $r=d(\tilde{p}, 0)=\log \frac{1+|\tilde{p}|}{1-\mid \tilde{p}} ;$ thus $|\tilde{p}|=\tanh (r / 2)$, with $\tilde{p}=\tilde{C}(p)$, if $p \in S$. Furthermore $\cosh \left(\frac{r}{2}\right)^{-2}=\frac{4 a}{\left(1+a+\frac{1}{4}|X|^{2}\right)^{2}+|Z|^{2}}$ and the image of the left Haar measure on $S$ via $\tilde{C}^{-1}$ is $d \mu=J(r) d \sigma d r$, where $r, \sigma$ are the radial coordinates on $B, r^{2}=|X|^{2}+|Z|^{2}+u^{2}$ and $J(r)=2^{p} \sinh (r / 2)^{p} \sinh (r)^{q}$ (see [2], Section 4).

The symmetric spaces of negative curvature are a main subclass of the DamekRicci spaces. Let $G$ be a connected, noncompact, semisimple Lie group of real rank one. Let $K$ be a maximal compact subgroup of $G$ and let $\mathfrak{g}$ and $\mathfrak{k}$ be the corresponding Lie algebras. If $G=N A K$ is an Iwasawa decomposition of $G$, then $N$ is an $H$-type group and $S=N A \approx G / K$ is a solvable Lie group in the class introduced above. Indeed, if $\mathfrak{a}$ and $\mathfrak{n}$ denote the Lie algebras of $A$ and $N$ respectively, $\mathfrak{n}$ splits $\mathfrak{n}=\mathfrak{g}_{\alpha / 2} \oplus \mathfrak{g}_{\alpha}$, where $\mathfrak{g}_{j \alpha}, j=1 / 2,1$, denote the $j \alpha$-root spaces of $\mathfrak{a}$. In the notation above we have $\mathfrak{n}_{\alpha}=\mathfrak{z}, \mathfrak{n}_{\alpha / 2}=\mathfrak{v}, \mathfrak{a}=\mathbf{R} H_{o}$, with $H_{o} \in \mathfrak{a}$ such that $\alpha\left(H_{o}\right)=1$. If on $S=N A$ we use the $G$-invariant metric induced by $2(p+4 q)^{-1} B$ ( $B$ the Killing form of $\mathfrak{g}$ ), then $S$ is isometric to a Damek-Ricci space. We note that, because of our convention, if $\mathfrak{n}$ is abelian, then $p=0, q=\operatorname{dim} \mathfrak{n}$.

\section{The Resolvent of the Laplacian on $S$}

Damek-Ricci spaces have strong similarities with symmetric spaces of negative curvature, in particular they are harmonic spaces. On $S$ there is a radialization operator $\pi$ which corresponds to the standard operator in the case of the ball model of $S$ (see [2], p. 230). If $f \in C_{c}^{\infty}(S), p \in S$ and $\tilde{p}=\tilde{C}(p)$ then

$$
\pi f(p):=\int_{S^{p+q}} \tilde{f}(\|\tilde{p}\| \sigma) d \sigma
$$

where $\tilde{f}:=f \circ \tilde{C}^{-1}$. In the symmetric case, if $f \in C^{\infty}(N A)$, then $\pi f(x)=$ $\int_{K} \bar{f}(k x) d k$, where $\bar{f}$ denotes the right $K$-invariant extension of $f$.

If $\left\{Z_{i}\right\},\left\{V_{j}\right\}$ are orthonormal bases of $\mathfrak{z}$ and $\mathfrak{v}$ respectively, the Laplace-Beltrami operator is given by $L=\sum_{i} Z_{i}^{2}+\sum_{j} V_{j}^{2}+H^{2}-Q H ; L$ generates the algebra of left-invariant differential operators on $S$ which commute with $\pi$ (see 2], Theorem $5.2)$.

If $f$ is a smooth radial function on $S-\{e\}$, we will often abuse notation by writing $f(r)=f(x)$, where $r=d(x, e)$. The action of $L$ on radial functions is given by

$$
L f(r)=\frac{d^{2}}{d r^{2}} f(r)+\frac{1}{2}(p \operatorname{coth}(r / 2)+2 q \operatorname{coth}(r)) \frac{d}{d r} f(r) .
$$


In the symmetric case, if $\mathfrak{n}$ is not abelian and we set $r=2 t$, then $L f(t)$ corresponds to $\frac{1}{4} C f\left(a_{t}\right), C$ the Casimir element; [6], Section 1 (1). If $\mathfrak{n}$ is abelian, then $L$ corresponds to $C$.

A spherical function $\psi$ on $S$ is a radial eigenfunction of $L$ such that $\psi(e)=1$. This generalizes the corresponding notion in the symmetric case and one has the following characterization $([2])$.

Proposition 2.1. Let $\nu \in \mathbf{C}$. The function $\phi_{\nu}=\pi\left(a^{\nu+Q / 2}\right)$ is a spherical function with eigenvalue $\lambda(\nu)=\nu^{2}-Q^{2} / 4$. Any spherical function on $S$ is of this form.

As in the symmetric case, we can express $\phi_{\nu}$ by a hypergeometric function as follows. By letting $z=-\sinh (r / 2)$, the equation

$$
\left\{\frac{d^{2}}{d r^{2}}+\frac{1}{2}(p \operatorname{coth}(r / 2)+2 q \operatorname{coth}(r)) \frac{d}{d r}-\lambda(\nu)\right\} f_{\nu}(r)=0
$$

transforms into the hypergeometric equation with parameters $a=Q / 2-\nu, b=$ $Q / 2+\nu$, and $c=n / 2$. Since $\phi_{\nu}(e)=1$, it follows that

$$
\phi_{\nu}(r)=F\left(-\nu+Q / 2, \nu+Q / 2, \frac{n}{2},-\sinh (r / 2)^{2}\right) .
$$

Furthermore, if $\operatorname{Re} \nu>0$, the asymptotic behavior of $\phi_{\nu}(r)$, as $r \rightarrow \infty$, is given by (see [2], p. 239)

$$
\phi_{\nu}(r) \sim c(\nu) e^{r(\nu+Q / 2)}, \quad \text { where } \quad c(\nu)=\frac{2^{-2 \nu+Q} \Gamma(n / 2) \Gamma(2 \nu)}{\Gamma(\nu+Q / 2) \Gamma\left(\nu+\frac{p+2}{4}\right)} .
$$

Here $c(\nu)$ coincides with Harish Chandra's $c$-function in the symmetric case. The Plancherel measure, $\mu(\nu)=(c(\nu) c(-\nu))^{-1}$, can be written $\mu(\nu)=c_{o} p(\nu) D(\nu), c_{o}$ a constant and $p(\nu)$ the polynomial given by

$$
\begin{array}{ll}
\prod_{j=0}^{\frac{p}{4}-1}\left(-\nu^{2}+\left((2 j+1)^{2} / 4\right)\right) \prod_{j=0}^{\frac{Q}{2}-1}\left(-\nu^{2}+\left(j^{2} / 4\right)\right), & q, \frac{p}{2} \text { even, } \\
-\prod_{j=1}^{p / 4}\left(-\nu^{2}+j^{2}\right)^{2} \nu^{3}, & q=1, \frac{p}{2} \text { odd }, \\
-\prod_{j=0}^{\frac{p}{4}-1}\left(-\nu^{2}+\left((2 j+1)^{2} / 4\right)\right) \prod_{j=0}^{\frac{Q}{2}-1}\left(-\nu^{2}+\left((2 j+1)^{2} / 4\right)\right) \nu, & q \text { odd }, \frac{p}{2} \text { even, }
\end{array}
$$

and $D(\nu)$ equals respectively $1, \cot (\pi \nu)$, and $\tan (\pi \nu)([1])$.

Remark. We note that $p$ is always even, since $\mathfrak{v}$ is a module over the Clifford algebra of $\mathfrak{z}$. If $p=0$, then $X \approx H^{q+1}, G \simeq \mathbf{S O}(q+1,1)$ and in this case $D(\nu)$ equals 1 or $\tan (\pi \nu)$ depending on whether $q$ is even or odd.

In [6], the resolvent of the Laplacian $R(\lambda(\nu))$ was studied on symmetric (and locally symmetric spaces) of negative curvature. In the symmetric case, it is given for $\operatorname{Re} \nu>\rho$ by convolution with a smooth radial function $Q_{\nu}$ on $S-\{e\}$ which is an eigenfunction of $L$ with eigenvalue $\lambda(\nu)$, and which has a meromorphic continuation to $\mathbf{C}$. As we shall now see, these properties remain valid for any $S$ as above. Many arguments in [6] can be adapted, so we shall omit several proofs. On the other hand, we shall show how to obtain $Q_{\nu}$ by using a series solution. We thank N. Wallach for useful discussions on this point, which helped us to simplify the original argument. 
If $b \in \mathbf{R}$ and $\delta>0$, let $\mathcal{S}_{b, \delta}=\{\nu: \operatorname{Re} \nu>b,|\nu+j|>\delta \forall j \in-\mathbf{N}: b \leq j\}$. That is, $\mathcal{S}_{b, \delta}=\{\nu: \operatorname{Re} \nu>b\}$, if $b \geq 0$, and $\mathcal{S}_{b, \delta}$ is a half plane with finitely many discs removed, centered at $-1,-2, \ldots,-k$, with $-k \geq b$, if $b<0$.

Theorem 2.2. If $\nu \in \mathbf{C}, 2 \nu \notin-\mathbf{N}$, then there exists a radial function $Q_{\nu} \in$ $C^{\infty}(S-\{e\})$ with the following properties:

(a) $(L-\lambda(\nu)) Q_{\nu}=0$. For each $x \in S, Q_{\nu}(x)$ is holomorphic for $\nu \notin-\frac{1}{2} \mathbf{N}$ and in $\nu \in \frac{1}{2} \mathbf{N}, Q_{\nu}(s)$ has at most a simple pole. Furthermore, for any $b \in \mathbf{R}, \delta, r_{o}>0$, there exists $K=K\left(b, \delta, r_{o}\right)$ such that $\left|Q_{\nu}(r)\right| \leq K$ for any $r \geq r_{o}, \nu \in \mathcal{S}_{b, \delta}$.

(b) Where defined, $\phi_{\nu}=c(-\nu) Q_{\nu}+c(\nu) Q_{-\nu}$.

(c) As $r \mapsto 0, Q_{\nu}(r) \sim d(\nu) r^{-p-q+1}|\log r|^{\delta_{p+q, 1}}$, for some meromorphic function $d(\nu)$ on $\mathbf{C}$, holomorphic if $2 \nu \notin-\mathbf{N}$.

(d) $\lim _{r \mapsto 0^{+}} J(r) \frac{d}{d t} Q_{\nu}(r)=-2 \nu c(\nu)$.

(e) If $f \in C_{c}^{\infty}(S)$ and $2 \nu \notin-\mathbf{N}$, then

$$
\int_{S} Q_{\nu}\left(x^{-1} y\right)(L-\lambda(\nu) I) f(y) d y=-2 \nu c(\nu) f(x) .
$$

Proof. We look for a solution of (4) of the form $q_{\nu}(r)=\sum_{j=0}^{\infty} a_{j}(\nu) e^{-(\nu+Q / 2+j) r}$. Substituting in (44) and using $\operatorname{coth}(r)=\frac{1+e^{-2 r}}{1-e^{-2 r}}$, we get that

$$
\begin{aligned}
& \sum_{j \geq 0}(Q+j)(2 \nu+Q+j) a_{j}(\nu) e^{-j r}+p \sum_{j \geq 1}(\nu+Q / 2+j+1) a_{j+1}(\nu) e^{-j r} \\
& +\sum_{j \geq 2}(j+2)(2 \nu+j+2) a_{j+2}(\nu) e^{-j r}=0 .
\end{aligned}
$$

Thus, the coefficients $a_{j}(\nu)$ must satisfy the recurrence relations

$$
a_{1}(\nu)=a_{0}(\nu) f_{-1}(\nu), \quad a_{j+2}(\nu)=a_{j+1}(\nu) f_{j}(\nu)+a_{j}(\nu) g_{j}(\nu),
$$

where $f_{j}(\nu)=p \frac{\nu+Q / 2+j+1}{(j+2)(2 \nu+j+2)}$ and $g_{j}(\nu)=\frac{(Q+j)(2 \nu+Q+j)}{(j+2)(2 \nu+j+2)}$, for $j \geq 0$.

We thus set $q_{\nu}(r)=e^{-(\nu+Q / 2) r} \sum_{j=0}^{\infty} a_{j}(\nu) e^{-j r}$, where $a_{0}=1$, and if $2 \nu \notin-\mathbf{N}$, then the $a_{j}(\nu)$ are given by (8) .

If $b \in \mathbf{R}, \delta>0$ and $\nu \in \mathcal{S}_{b, \delta}$, we have

$$
\begin{gathered}
\left|f_{j}(\nu)\right| \leq \frac{p}{2 j+4}\left(1+\frac{Q+j}{|2 \nu+j+2|}\right) \leq \frac{p}{2 j+4}\left(1+\frac{Q+j}{(j+2-2 k)}\right), \\
\left|g_{j}(\nu)\right| \leq \frac{Q+j}{2+j}\left(1+\frac{|Q-2|}{|2 \nu+j+2|}\right) \leq \frac{Q+j}{j+2}\left(1+\frac{|Q-2|}{(j+2-2 k)}\right)
\end{gathered}
$$

for $j+2>2|k|$, where $k$ is the first integer such that $k \leq b$. These estimates clearly imply that given $\varepsilon>0$ there exist $j_{0}$ and $M=M(\varepsilon)$ such that $\left|f_{j}(\nu)\right| \leq \varepsilon,\left|g_{j}(\nu)\right| \leq$ $1+\varepsilon$, if $j \geq j_{0},\left|f_{j}(\nu)\right| \leq M,\left|g_{j}(\nu)\right| \leq M$, if $j<j_{0}$, uniformly for $\nu \in \mathcal{S}_{b, \delta}$. Using these estimates we see that if $\nu \in \mathcal{S}_{b, \delta}$, if $M^{\prime}=M^{\prime}(\varepsilon)=j_{0} M^{j_{0}}$, then

$$
\left|a_{j}(\nu)\right| \leq \begin{cases}j M^{j} & j \leq j_{0} \\ M^{\prime}(1+2 \varepsilon)^{j-j_{0}+1} & j \geq j_{0}\end{cases}
$$

Now, by (9) $\left|q_{\nu}(r)\right| \leq e^{-\left(\operatorname{Re} \nu+\frac{Q}{2}\right) r} M^{\prime}\left(j_{0}+\sum_{l \geq 0}(1+2 \varepsilon)^{l+1} e^{-\left(l+j_{0}\right) r}\right)$; hence the series defining $q_{\nu}$ converges absolutely and uniformly for $\nu \in \mathcal{S}_{b, \delta}$ and $r>r_{o}$, for 
each $r_{o}>0$. Since $r_{o}$ is arbitrary, $q_{\nu}$ defines a uniformly bounded function for $\nu, r$ in this region. With a similar argument one proves the uniform convergence of the series of the derivatives, in each region $\mathcal{S}_{b, \delta}, r>r_{o}$; hence $q_{\nu}$ is smooth. If we now define $Q_{\nu}(x)=q_{\nu}(r)$ with $r=d(x, e)$, for $x \in S$, then $Q_{\nu} \in C^{\infty}(S-\{e\})$ is a radial eigenfunction of $L$ of eigenvalue $\lambda(\nu)$ and has the properties stated in (a).

From now on we shall write $Q_{\nu}(r)=q_{\nu}(r)$, for simplicity. By the asymptotic behavior as $r \mapsto+\infty$, it follows that if $2 \nu \notin \mathbf{Z}, Q_{\nu}(r), Q_{-\nu}(r)$ form a fundamental system of solutions of (4). Writing $\phi_{\nu}$ in terms of $Q_{\nu}$ and $Q_{-\nu}$, the functional equation in (b) follows as in the symmetric case (see [6], p. 671).

We now prove (c). Equation (4) has a regular singular point at $r=0$ and the corresponding indicial equation is $s(s-1)+(p+q) s=0$, with roots $s=0$, $s=1-p-q$. The solution $\phi_{\nu}(r)$ is associated to the root $s=0$ and is continuous at $r=0$. If $2 \nu \notin-\mathbf{N}$, and if $p+q>1, Q_{\nu}$ is a second linearly independent solution; hence $\lim _{r \mapsto 0^{+}} Q_{\nu}(r) r^{p+q-1}:=d(\nu)$ exists and the meromorphy of $Q_{\nu}$ implies that of $d(\nu)$. Similarly, if $p+q=1, Q_{\nu}(r) \sim d(\nu) \log r$ as $r \mapsto 0^{+}$. Thus (c) follows. The proof of (d) is similar to that of [6], Lemma 1.3, and will be omitted.

To see (e) we may assume that $x=e$. We have, for any $f \in C_{c}^{\infty}(S)$,

$$
\begin{aligned}
\int_{S} Q_{\nu}(y)(L-\lambda(\nu) I) f(y) d \mu(y) & =\int_{\partial B} \int_{0}^{\infty} \tilde{Q}_{\nu}(r \sigma)(L-\lambda(\nu) I) \tilde{f}(r \sigma) J(r) d r d \sigma \\
& =\int_{0}^{\infty} Q_{\nu}(r) J(r)(L-\lambda(\nu)) \pi f(r) d r .
\end{aligned}
$$

Now we observe that for a radial function $h$ on $S, J(r)^{1 / 2} L h(r)=\frac{d^{2}}{d r^{2}} J^{1 / 2}(r) h(r)+$ $J(r)^{1 / 2} \eta(r) h(r)$, where $\eta=\frac{\left(J^{\prime}\right)^{2}-2 J^{\prime \prime} J}{4 J^{2}}$. Hence we see that the above equals

$$
\begin{aligned}
& \int_{0}^{\infty} \frac{d^{2}}{d r^{2}}\left(J(r)^{1 / 2} \pi f(r)\right) J(r)^{1 / 2} Q_{\nu}(r)-J(r)^{1 / 2} \pi f(r) \frac{d^{2}}{d r^{2}}\left(J(r)^{1 / 2} Q_{\nu}(r)\right) d r \\
= & \int_{0}^{\infty} \frac{d}{d r}\left[\frac{d}{d r}\left(J(r)^{1 / 2} \pi f(r)\right) J(r)^{1 / 2} Q_{\nu}(r)-J(r)^{1 / 2} \pi f(r) \frac{d}{d r}\left(J(r)^{1 / 2} Q_{\nu}(r)\right)\right] d r \\
= & -2 \nu c(\nu) f(e)
\end{aligned}
$$

using (c) and (d). This gives (e); hence the theorem follows.

\section{The RESIDUes OF THE RESOlvent}

Let $\tilde{R}(\lambda(\nu))$ denote the kernel operator with kernel $K_{\nu}(x, y)=-\frac{Q_{\nu}\left(x^{-1} y\right)}{2 \nu c(\nu)}$. If $\operatorname{Re} \nu>\rho$, then $\tilde{R}(\lambda(\nu))=R(\lambda(\nu))$.

Theorem 3.1. If $p, q$ are both even, then $\tilde{R}(\lambda(\nu))$ is everywhere holomorphic. Otherwise, it has simple poles lying at $\nu_{k}=-Q / 2-k$ with $k \in \mathbf{N} \cup\{0\}$. If $\nu=\nu_{k}$, set $T_{\nu_{k}}(f):=\operatorname{Res}_{\nu=\nu_{k}} \tilde{R}(\lambda(\nu))(f)$. Then $T_{\nu_{k}}(f)=\left(2 \pi \nu_{k}\right)^{-1} p\left(\nu_{k}\right) f * \dot{\phi}_{\nu}$ and $T_{\nu_{k}}$ is a finite rank operator, for each value of $k$.

Proof. The possible poles of $K_{\nu}(x, y)$ lie at $-\frac{1}{2} \mathbf{N}$ or at the zeros of $c(\nu)$. By using formula (6) one sees that $c(\nu)$ has no zeros in $\mathbf{C}$, if $p$ and $q$ are both even. Otherwise, $q$ is odd and $c(\nu)$ has simple zeros at $\nu_{k}=-Q / 2-k$, for any $k \in \mathbf{N} \cup\{0\}$, and possibly simple poles at $\nu \in-\frac{1}{2} \mathbf{N}$. 
Since $\nu=0$ is a simple pole of $c(\nu)$, and $Q_{\nu}$ is holomorphic at $0, \frac{Q_{\nu}}{2 \nu c(\nu)}$ is holomorphic at $\nu=0$.

On the other hand $c(-\nu)$ and $Q_{-\nu}$ are holomorphic and nonvanishing on $\mathbf{R}^{<0}$, $\phi_{\nu}$ is everywhere holomorphic and $\phi_{\nu}(1)=1$. So Theorem 2.2 (b) implies that a pole of $Q_{\nu}$ must be compensated by a pole of $c(\nu)$ and a zero of $c(\nu)$ cannot be a zero of $Q_{\nu}$.

Therefore, $\frac{Q_{\nu}}{2 \nu c(\nu)}$ has a pole at $\nu$ if and only if $\nu$ is a zero of $c(\nu)$, that is, $\nu=\nu_{k}=-Q / 2-k, k \in \mathbf{N} \cup\{0\}$. On the other hand, $\frac{Q_{-\nu}}{2 \nu c(-\nu)}$ is analytic at $\nu=\nu_{k}$. Thus, if $f \in C_{c}^{\infty}(S)$ and using that $-\frac{Q_{\nu}}{2 \nu c(\nu)}=\frac{Q_{-\nu}}{2 \nu c(-\nu)}-\frac{\mu(\nu) \phi_{\nu}}{2 \nu}$, we have

$$
T_{\nu_{k}}(f)=\operatorname{Res}_{\nu=\nu_{k}} \tilde{R}(\lambda(\nu))(f)=\frac{p\left(\nu_{k}\right)}{2 \pi \nu_{k}} f * \check{\phi}_{\nu_{k}} .
$$

From (5) and the expression for $\cosh \left(\frac{r}{2}\right)$ in the Preliminaries, we have that

$$
\phi_{\nu}(X, Z, a)=\sum_{i \geq 0} \frac{(Q / 2-\nu)_{i}(Q / 2+\nu)_{i}}{i !(n / 2)_{i}}\left[\frac{\left(a+\frac{1}{4}|X|^{2}\right)^{2}+|Z|^{2}}{4 a}\right]^{i},
$$

where $(u)_{i}=\prod_{l=0}^{i-1} u+l$ for $u \in \mathbf{C}$. Hence we see that the coefficients in the expansion (11) are zero for $i \geq k+1$, for the special values $\nu_{k}=-Q / 2-k$. Fix $\left\{V_{i}\right\}$ and $\left\{W_{j}\right\}$, orthonormal bases of $\mathfrak{v}$ and $\mathfrak{z}$ respectively, and write $X=\sum_{i=1}^{p} x_{i} V_{i}$ and $Z=\sum_{j=1}^{q} z_{j} W_{j}$. If $I=\left(i_{1}, \ldots, i_{p}\right), J=\left(j_{1}, \ldots, j_{q}\right)$, set $X^{I}=\prod x_{j}^{i_{j}}, Z^{J}=\prod z_{l}^{j_{l}},|I|=\sum_{1}^{p} i_{j}$ and similarly for $|J|$. Let $\mathcal{F}_{k}$ be the linear span of the functions $a^{i} X^{2 I} Z^{J}: i \in \mathbf{Z}$, $|i| \leq k,|I|,|J| \leq 2 k$. Clearly $\phi_{\nu_{k}} \in \mathcal{F}_{k}$. If $t=(Y, U, b)$ with $Y=\sum_{i=1}^{p} y_{i} V_{i} \in \mathfrak{v}$, $U=\sum_{i=1}^{q} u_{i} W_{i} \in \mathfrak{z}, b \in A$ and $s=(X, Z, a) \in S$, then

$$
\begin{aligned}
t^{-1} s & =\left(b^{-\frac{1}{2}}(X-Y), b^{-1}\left(Z-U+\frac{1}{2}[X, Y]\right), b^{-1} a\right) \\
& =\left(b^{-\frac{1}{2}} \sum\left(x_{i}-y_{i}\right) V_{i}, b^{-1} \sum\left(z_{j}-u_{j}\right) W_{j}+\frac{1}{2} \sum_{l} \sum_{i, j} x_{i} y_{j} a_{i, j}^{l} W_{l}, b^{-1} a\right),
\end{aligned}
$$

where $[X, Y]=\sum_{l} \sum_{i, j} x_{i} y_{j} a_{i, j}^{l} W_{l}$. Hence, by (11) $\phi_{\nu_{k}}\left(t^{-1} s\right)$ is a linear combination of functions of the form $a^{j_{1}} b^{j_{2}} X^{2 I_{1}} Y^{2 I_{2}} Z^{J_{1}} U^{J_{2}}$ with $j_{i} \in \mathbf{Z},\left|j_{i}\right| \leq k, i=1,2$, and $\left|I_{i}\right|,\left|J_{i}\right| \leq 2 k$ for $i=1,2$.

Therefore, if $f \in C_{c}^{\infty}(S)$, it follows that $f * \check{\phi}_{\nu_{k}}(t)=\int_{S} f(s) \phi_{\nu_{k}}\left(t^{-1} s\right) d s$ is a linear combination of expressions of the form

$$
t \mapsto b^{j_{2}} Y^{2 I_{2}} U^{J_{2}} \int_{\mathfrak{z}} \int_{\mathfrak{v}} \int_{A} f(X, Z, a) a^{j_{1}} X^{2 I_{1}} Z^{J_{1}} a^{-Q-1} d a d X d Z .
$$

Therefore, $f * \check{\phi}_{\nu_{k}}$ belongs to $\mathcal{F}_{k}$, a finite dimensional space, as asserted. 


\section{The SYMmETRIC CASE}

In the case when $S$ is of symmetric type one can get more precise information on the operators $T_{\nu_{k}}$ by using representation theory.

The group of isometries $G$ of $S$ is a noncompact semisimple Lie group of real rank one. Let $\mathfrak{g}, \mathfrak{k}, N$, and $A$ be as in Section 1 , let $M$ be the centralizer of $A$ in $K$, let $P=M A N$ and let $\mathfrak{p}$ be the Lie algebra of $P$. Extend $\mathfrak{a}$ in the usual way to a Cartan subalgebra $\mathfrak{h}_{c}=\mathfrak{a}_{c}+\mathfrak{h}_{c}^{-}$of $\mathfrak{g}$, where $\mathfrak{h}^{-}$is a maximal abelian subalgebra of $\mathfrak{m}$, and introduce compatible orderings in the dual spaces of $\mathfrak{a}$ and $\mathfrak{a}+\sqrt{-1} \mathfrak{h}^{-}$. Let $\Sigma^{+}\left(\Delta^{+}\right)$denote the corresponding set of positive roots of the pair $(\mathfrak{g}, \mathfrak{a})$ (respectively $\left(\mathfrak{g}_{c}, \mathfrak{h}_{c}\right)$ ). Since $\mathfrak{g}$ has real rank one, there is only one real root $\tilde{\alpha} \in \Delta^{+}$. It satisfies $\tilde{\alpha}_{\mid \mathfrak{h}^{-}}=0$ and $\tilde{\alpha}_{\mid \mathfrak{a}}=\alpha$.

For $\nu \in \mathbf{C}$, let $\left(\pi_{\nu}, H^{\nu}\right)$ be the spherical principal series representation of $G$ (see 7], Section 3.6). The zonal spherical function $\phi_{\nu}$ is given by $\phi_{\nu}(g)=\left\langle\pi_{\nu}(g) 1_{\nu}, 1_{\nu}\right\rangle$, where $1_{\nu} \in H^{\nu}$ is such that $1_{\nu}(n a k)=a^{(\nu+\rho) \alpha}, n \in N, a \in A, k \in K$, and $\langle$,$\rangle is$ the standard inner product on $H^{\nu}$.

Theorem 4.1. Let $S=G / K$ be a noncompact symmetric space of real rank one and let $\nu_{k}=-\rho-k$ with $k \in \mathbf{N} \cup\{0\}$. Then $\operatorname{Im}\left(T_{\nu_{k}}\right)$ is an irreducible $\mathfrak{g}_{c}$-module of highest weight $k \tilde{\alpha}$.

Proof. By a result of Helgason (see 4, Ch. V, Theorem 4.1), the $K$-spherical finite dimensional representations of $G$ can be characterized as the representations of $\mathfrak{g}_{c}$ of highest weight $\Lambda \in \mathfrak{h}_{c}^{*}$ such that: $\Lambda_{\mid \mathfrak{h}^{-}}=0$ and $\langle\Lambda, \lambda\rangle /\langle\lambda, \lambda\rangle \in \mathbf{Z}^{\geq 0}$, for any $\lambda \in \Sigma^{+}$. Since in our case $\Sigma^{+}=\{\alpha, \alpha / 2\}$ or $\{\alpha\}$, this is equivalent to $\Lambda_{\mid \mathfrak{a}}=k \tilde{\alpha}$, with $k \in \mathbf{Z}^{\geq 0}$, and $\tilde{\alpha}$ the real root. We shall denote by $V_{k \tilde{\alpha}}$ the $\mathfrak{g}_{c}$-module with highest weight $k \tilde{\alpha}$.

Our claim is that $1_{\nu_{k}}$ generates a finite dimensional $(\mathfrak{g}, K)$-submodule $V_{\nu_{k}}$ of $H^{\nu_{k}}$, isomorphic to $V_{k \tilde{\alpha}}$.

In the notation of Lemma 3.8.2 in [7], we have that

$$
\operatorname{Hom}_{\mathfrak{g}, K}\left(V_{k \tilde{\alpha}}, H^{\nu_{k}}\right) \simeq \operatorname{Hom}_{\mathfrak{p}, M}\left(V_{k \tilde{\alpha}} / \mathfrak{n} V_{k \tilde{\alpha}}, \mathbf{C}_{\nu_{k}}\right),
$$

where $\mathbf{C}_{\nu_{k}}$ denotes the $M A N$-module $\mathbf{C}$, with $M N$ acting trivially and $a \in A$ acting by multiplication by $a^{\left(\nu_{k}+\rho\right) \alpha}$. To prove our claim it will thus be sufficient to show that there exists a nontrivial $(\mathfrak{p}, M)$-morphism $f: V_{k \tilde{\alpha}} / \mathfrak{n} V_{k \tilde{\alpha}} \rightarrow \mathbf{C}_{\nu_{k}}$. We denote by $\Lambda_{o}$ the lowest weight of $V_{k \alpha}$ and by $v_{o}$ the corresponding lowest weight vector. Then $\Lambda_{o}=s_{o} \Lambda, s_{o}$ the long element of the Weyl group of $\left(\mathfrak{g}_{c}, \mathfrak{h}_{c}\right)$. Since $\Lambda^{\prime}=-s_{o} \Lambda$ is the highest weight of the dual representation of $V_{k \tilde{\alpha}}$, which is also $K$-spherical, $\Lambda^{\prime}$ satisfies Helgason's conditions. This implies that $s_{o} \Lambda_{\mid \mathfrak{h}-}=0$ and $s_{o} \Lambda_{\mid \mathfrak{a}}=-k \alpha$. Arguing as in the proof of Theorem 4.1, Ch. V in 4], one shows that $\pi_{\Lambda}(M) v_{o}=v_{o}$.

Since $s_{o} \Lambda_{\mid \mathfrak{h}^{-}}=0$, it follows that $V=\mathbf{C} v_{o} \oplus(\mathfrak{n} \oplus \mathfrak{m}) V$. Now we can define a $(\mathfrak{p}, M)$-morphism $f: V / \mathfrak{n} V \rightarrow \mathbf{C}_{\nu}$ such that $f:\left[v_{o}\right] \mapsto 1$, where $\left[v_{o}\right]$ is the class of $v_{o}$ and $f=0$ on $\mathfrak{m} V$. Hence, by $\left(\underline{12}\right.$, there is a nonzero $G$-map of $V_{k \tilde{\alpha}}$ onto a subspace $V_{\nu_{k}}$ of $H^{\nu_{k}}$, which must contain $1_{\nu_{k}}$.

Now we prove the statement in the theorem. If $f \in C_{c}^{\infty}(G / K)$, and $x \in G$, we have by 3.1

$$
T_{\nu_{k}}(f)=p_{k} f * \check{\phi}_{\nu_{k}}(x)=p_{k}\left\langle\pi\left(x^{-1}\right) \pi(f) 1_{\nu_{k}}, 1_{\nu_{k}}\right\rangle,
$$

where $p_{k}=-\frac{p\left(\nu_{k}\right)}{\pi \nu_{k}} \neq 0$, for all $k$ (see the formula of $p(\nu)$ in Section 2). 
By irreducibility, as $f$ varies, $\pi(f) 1_{\nu_{k}}$ fills $V_{\nu_{k}} \simeq V_{k \alpha}$. Hence, the image of $T_{\nu_{k}}$ coincides with the image of the $G$-morphism $T_{k}: V_{\nu_{k}} \mapsto C^{\infty}(G / K)$ given by $T_{k}(v)(x)=\left\langle\pi_{\nu_{k}}\left(x^{-1}\right) v, 1_{\nu}\right\rangle$, for $v \in V_{\nu_{k}}$. This proves the theorem.

Remark 4.2. We will now use the Weyl dimension formula to calculate the dimension of the $\mathfrak{g}_{c}$-module $V_{k \tilde{\alpha}}$ in each case. The real roots $\tilde{\alpha}$ can be read from the Satake diagram of $\mathfrak{g}$. They are listed, for each rank one group, in [5] (for instance). We shall thus use the notation in [5].

(i) $\mathfrak{g}=\mathfrak{s o}(\mathbf{n}, \mathbf{1})$ ( $n$ even). In this case, the real root is $\tilde{\alpha}=\epsilon_{1}$, the first fundamental weight. The corresponding $\mathfrak{g}_{c}$-module $V_{k \tilde{\alpha}}$ is isomorphic to the representation of $G$ on $\mathcal{H}_{k}$, the space of homogeneous harmonic polynomials of degree $k$ in $n+1$ variables, which has dimension $\frac{(k+n-2) !(2 k+n-1)}{k !(n-1) !}$. This can easily be computed by the Weyl dimension formula.

(ii) $\mathfrak{g}=\mathfrak{s} \mathbf{u}(\mathbf{n}, \mathbf{1})$. Here, the real root is $\tilde{\alpha}=\epsilon_{1}-\epsilon_{n+1}$, and the positive roots are $\epsilon_{i}-\epsilon_{j}, i<j$ and $2 \rho=\sum_{j=1}^{n+1}(n-2 j+2) \epsilon_{j}$. Thus

$$
\begin{aligned}
& \operatorname{dim}\left(V_{k \tilde{\alpha}}\right)= \prod_{1 \leq i<j \leq n+1} \frac{\left\langle k\left(\epsilon_{1}-\epsilon_{n+1}\right)+\rho, \epsilon_{i}-\epsilon_{j}\right\rangle}{\left\langle\rho, \epsilon_{i}-\epsilon_{j}\right\rangle} \\
&=\prod_{2 \leq j \leq n} \frac{k+j-1}{j-1} \prod_{2 \leq i \leq n} \frac{k+n+1-i}{n+1-i} \frac{2 k+n}{n}=\left(\begin{array}{c}
k+n-1 \\
k
\end{array}\right)^{2} \frac{2 k+n}{n} .
\end{aligned}
$$

(iii) $\mathfrak{g}=\mathfrak{s p}(\mathbf{n}, \mathbf{1})$. In this case, $\tilde{\alpha}=\epsilon_{1}+\epsilon_{2}$, and the positive roots are $\epsilon_{i} \pm \epsilon_{j}, 1 \leq$ $i<j \leq n+1$, and $2 \epsilon_{i}, 1 \leq i \leq n+1$. Also, $\rho=\sum_{j=1}^{n+1}(n+2-j) \epsilon_{j}$. Hence

$$
\begin{aligned}
\operatorname{dim}\left(V_{k \tilde{\alpha}}\right) & =\left(\prod_{i=1}^{2} \prod_{j=3}^{n+1} \frac{2 n+4-j-i+k}{2 n+4-j-i} \cdot \frac{j-i+k}{j-i}\right) \frac{2 n+1+2 k}{2 n+1} \cdot \frac{n+k}{n} \cdot \frac{n+k+1}{n+1} \\
& =\left(\begin{array}{c}
2 n+k-1 \\
k
\end{array}\right)^{2} \frac{2 n+k}{(2 n+1)(2 n)} \frac{2 n+2 k+1}{k+1}
\end{aligned}
$$

(iv) $\mathfrak{g}=\mathfrak{f}_{4}$. The real root is $\tilde{\alpha}=\lambda_{4}\left(=\epsilon_{1}\right)$, the fourth fundamental weight. The positive roots are $\epsilon_{i}, \epsilon_{i} \pm \epsilon_{j}, 1 \leq i<j \leq 4, \frac{1}{2}\left(\epsilon_{1} \pm \epsilon_{2} \pm \epsilon_{3} \pm \epsilon_{4}\right)$ and $2 \rho=$ $11 \epsilon_{1}+5 \epsilon_{2}+3 \epsilon_{3}+\epsilon_{4}$. Using the Weyl dimension formula we obtain in this case

$$
\operatorname{dim}\left(V_{k \tilde{\alpha}}\right)=\frac{2 k+11}{11} \prod_{j=1}^{j=10} \frac{k+j}{j} \cdot \prod_{j=4}^{j=7} \frac{k+j}{j} .
$$

4.1. The real hyperbolic $n$-space. If $S \approx H^{n}$, one can make the results in Theorem 2.2 more precise. In this case one can solve the recurrence in (8), obtaining an explicit series expression for $Q_{\nu}$. Indeed, since $p=0$, by (8) we see that $a_{2 j+1}=0$ for $j \geq 0$; hence $a_{2 j}=a_{2 j-2} \frac{(j-1+\rho)(j-1+\rho+\nu)}{j(\nu+j)}, j \geq 0$. Thus, if we set $c_{j}:=a_{2 j}$, for $j \geq 0$, and if $c_{0}:=1$, we obtain for $j \geq 1$

$$
c_{j}(\nu)=\frac{(\rho)_{j}}{j !} \frac{(\nu+\rho)_{j}}{(\nu+1)_{j}}
$$


Furthermore, $c(\nu)=\frac{2^{2 \rho-1} \Gamma(n / 2)}{\pi^{1 / 2}} \frac{\Gamma(\nu)}{\Gamma(\nu+\rho)}$ hence, using (13) and the duplication formula for the Gamma function, we obtain

$$
\frac{Q_{\nu}(r)}{2 \nu c(\nu)}=\frac{2^{-2 n+3}}{(n-2) !} e^{-(\nu+\rho) r} \sum_{j=0}^{\infty} \frac{\Gamma(\rho+j)}{j !} \frac{\Gamma(\nu+\rho+j)}{\Gamma(\nu+j+1)} e^{-2 j r}
$$

Now, if $S_{b, \delta}$ is as in Theorem 2.2, one sees, by using Stirling's estimates, that there exists a constant $K=K(b, \delta)$ such that the coefficients in (14) are bounded by $K j^{\rho-1}|\nu+j|^{\rho-1}$, uniformly for $\nu$ in $S_{b, \delta}$. This gives an alternative proof of the convergence, as stated in Theorem 2.2 (a).

Regarding the poles, we see that if $n$ is odd, since $\rho=\frac{n-1}{2} \in \mathbf{N}$, the coefficients in (14) are polynomial functions in $\nu$; hence $\tilde{R}(\lambda(\nu))$ is everywhere holomorphic in this case.

If $n$ is even, (14) implies that the kernel is meromorphic with poles at $\nu_{k}=$ $-\rho-k, k \in \mathbf{N} \cup\{0\}$. Since $\Gamma(\nu+\rho+j)$ is holomorphic at $\nu=\nu_{k}$ for $j>k$, we get

$$
\begin{aligned}
\operatorname{Res}_{\nu=\nu_{k}} \frac{Q_{\nu}(r)}{2 \nu c(\nu)} & =\frac{2^{-2 n+3}}{(n-2) !} \sum_{j=0}^{k} \frac{\Gamma(\rho+j)(-1)^{k-j}}{j !(k-j) ! \Gamma(-k-\rho+j+1)} e^{-(2 j-k) r} \\
& =\frac{2^{-2 n+4}}{(n-2) !} \sum_{j=0}^{[k / 2]} \frac{\Gamma(\rho+j)(-1)^{k-j}}{j !(k-j) ! \Gamma(-k-\rho+j+1)} \cosh (2 j-k) r,
\end{aligned}
$$

since $\frac{\Gamma(\rho+j)(-1)^{k}}{\Gamma(-k-\rho+j+1)}=\frac{\Gamma(\rho+k-j)}{\Gamma(-\rho-j+1)}$, for $0 \leq j \leq k$.

Remark 4.3. We note that in [3, Section 2, Guillopé-Zworski consider the resolvent kernel for the real hyperbolic $n$-space, giving the location of the poles and showing that the residues define operators of finite rank.

The authors wish to thank Fulvio Ricci for useful conversations on an earlier version of this paper.

\section{REFERENCES}

[1] Anker J.P., Damek E., Yacoub Ch., Spherical analysis on harmonic AN groups, Annali della Scuola Normale Sup. di Pisa 23 (1996), 643-679. MR 99a:22014

[2] DameK E., Ricci F., Harmonic analysis on solvable extensions of $H$-type groups, Journal of Geometric Analysis 2 (1992), 213-248. MR 93d:43006

[3] Guillopé L., Zworski M., Polynomial bounds for the number of resonances for some complete spaces of constant negative curvature near infinity, Asymptotic Analysis 11 (1995), 1-22.

[4] Helgason S., Groups and Geometric Analysis, Pure and Applied Math 113, Academic Press, 1984. MR 86c:22017

[5] Miatello R.J., On the Plancherel measure for linear Lie groups of rank one, Manuscripta Math. 29 (1979), 249-276. MR 80h:22021

[6] Miatello R.J., Wallach N.R., The resolvent of the Laplacian on negatively curved locally symmetric spaces of finite volume, Jour. Diff. Geometry 36 (1992), 663-698. MR 93i:58160

[7] Wallach N. R., Real Reductive Groups I, Pure and Applied Math 132, Academic Press, 1988. MR 89i:22029

Facultad de Matemática, Astronomía y Física, Universidad Nacional de Córdoba, 5000 Córdoba, Argentina

E-mail address: miatello@mate.uncor.edu

E-mail address: cwill@mate.uncor.edu 